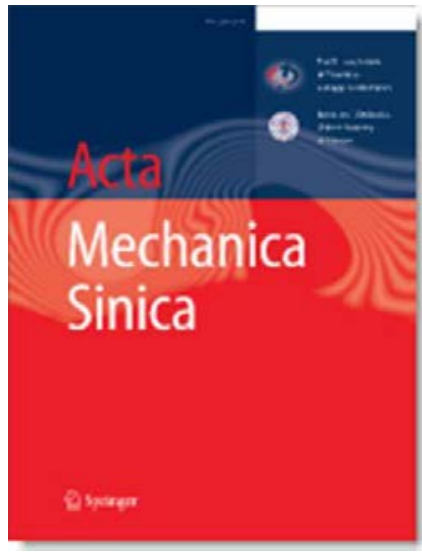

This is a preliminary PDF of the author-produced manuscript that has been peer-reviewed and accepted for publication in Acta Mechanica Sinica (AMS), since it is being posted soon after acceptance, it has not yet been formatted, or processed by AMS Publications. This preliminary version of the manuscript may be downloaded, distributed, and cited, but please be aware that there will be visual differences and possibly some content differences between this version and the final published version.

The DOI for this manuscript is doi: .

Please use the following full citation:

Chen, Q., Sun, F., Li, Z.Y., Taxis, L., Pugno, N.: Mechanics of a ring structure subjected to multi-pairs of evenly distributed equal radial forces. Acta Mechanica Sinica., doi: , in press. 


\title{
Mechanics of a ring structure subjected to multi-pairs of evenly distributed equal radial forces
}

\author{
Q. Chen ${ }^{1, *}$, F. Sun ${ }^{2}$, Z.Y. Li $^{1,3}$, L. Taxis ${ }^{4}$, N. Pugno ${ }^{4,5,6}$
}

1. Biomechanics Laboratory, School of Biological Science and Medical Engineering, Southeast University, Nanjing 210096, P.R. China

2. State Key Laboratory of Mechanics and Control of Mechanical Structures, Nanjing University of Aeronautics and Astronautics, Nanjing 210096, P.R. China.

3. School of Chemistry, Physics and Mechanical Engineering, Queensland University of Technology (QUT), Brisbane QLD 4001, Australia

4. Laboratory of Bio-Inspired and Graphene Nanomechanics, Department of Civil, Environmental and Mechanical Engineering, University of Trento, Trento 38123, Italy

5. School of Engineering and Materials Science, Queen Mary University of London, Mile End Road E14NS, London, UK

6. Ket Lab, Italian Space Agency, Via del Politecnico snc, Rome 00133, Italy.

*Corresponding Author:

Dr. Qiang CHEN

Email: chenq999@gmail.com

Tel./Fax: $+\underline{+862583792620}$ 
Abstract Combining the elastica theory, finite element (FE) analysis, and a geometrical topological experiment, we studied the mechanical behavior of a ring subjected to multi-pairs of evenly distributed equal radial forces by looking at its seven distinct states. The results showed that the theoretical predictions of the ring deformation and strain energy matched the FE results very well, and that the ring deformations were comparable to the topological experiment. Moreover, no matter whether the ring was compressed or tensioned by $N$-pairs of forces, the ring always tended to be regular polygons with $2 N$ sides as the force increased, and a proper compressive force deformed the ring into exquisite flower-like patterns. The present study solves a basic mechanical problem of a ring subjected to lateral forces, which can be useful for studying the relevant mechanical behavior of ring structures from the nano- to the macro-scale.

Keyword Ring structures, Mechanical properties, Shape, Strain energy. 


\section{INTRODUCTION}

Ring structures are very common all the way from the nano-scale up to the macro-scale. Such structures include 2D rings formed from bars, e.g. 2D carbon-nanorings [1], and 3D rings (or cylindrical tubes) formed from thin plates, e.g. 3D steel/aluminum tubes [2]. They generally exhibit variable geometries under different lateral loading conditions, and their mechanical behaviors under conditions such as buckling [1], collapse [2] and impact dynamics [3] etc., have been studied. Carbon nanorings, for example, become elliptical before the buckling of the carbon nanotube [1], and cross-sections of metallic tubes deformed inelastically become double-symmetric $[2,3]$ with plastic hinges. Thus, understanding mechanics of ring structures under lateral loads is beneficial to the present scientific community.

2D rings subjected to lateral loads were studied to understand their stress distribution [4-6], in-plane free vibration [7,8] and impact/rebound [3]. Plus, considering size effect, the free vibration of 2D nanorings under lateral loads was also investigated [9,10]. In particular, Chen et al. [11] studied a connected carbon nanoring, which was found to have a great Young's modulus, and then, based on this work, Feng and Liew [1] studied the critical buckling displacement and shape of the laterally tensioned basic element—a single carbon nanoring. However, the studies on the mechanical behavior of the carbon nanoring were based on molecular mechanics or molecular dynamics.

To the best knowledge of the authors, 3D ring structures subjected to axial loads have been extensively studied due to their fascinating properties, such as buckling patterns and energy absorption of cylindrical shells [12-14], but their mechanical properties under lateral forces have rarely been studied. In this regard, a single tube was laterally compressed by Ghosh et al. [15], as 
an energy absorber, and the mechanical behavior of round nested metallic tubes systems (i.e., combined cylindrical tubes) between two rigid plates, has been studied [16,17], also as an energy absorbers. The quasi-static lateral compression, post-collapse and crushing behavior of the tube systems were experimentally and numerically analyzed, and later reviewed by Olabi et al. [18].

The above literature focused on a ring subjected to a single lateral concentrated force or a pair of lateral forces along a diameter. Also, there are multi-pairs of concentrated forces centrally acting on a ring, which often exist in the form of a basic element in a complex structure, for example, circular joints subjected to two or three pairs of lateral forces in the centre-symmetric tetragonal or hexagonal honeycombs [19]. Thus, in views of rings subjected to few pairs of centrally acting forces along diameters [17,19], we here extend them to a general case, for which a single ring is subjected to multi-pairs of equal, radial, concentrated forces evenly distributed around the ring, and find the load-deformation or load-strain energy relationships.

\section{THEORETICAL MODELS}

The elastica theory is always employed to characterize very large deformations of curved structures, where the shear and axial deformations are not considered, for example, it is used to study a micro-beam, droplet, CNT ring adhered on a substrate [20], and interestingly, an island-bridge model in a stretchable electronic, in which the large deformation of the bridge structure was modeled as an elastica beam with both ends fixed [21]. However, it is noticed that real curved structures are not elastica, and they usually deform plastically when subjected to large loads [2,3], plus, the shear and axial deformations cannot be neglected when the bar or plate forming the curved structures is thick, otherwise, the Timoshenko beam theory has to be employed, 
in this case, it reduces the bending rigidity of the curved structures due to the involvement of the shear effect, and improves the structural deformation. Here, the theory for thin structures is used to study the ring elastica under multi-pairs of equal forces acting along diameters, and in-plane/out-of-plane stability is not considered. To comprehensively describe the ring's mechanical behaviors, the ring under both compression and tension are treated, and the compressive case comes first.
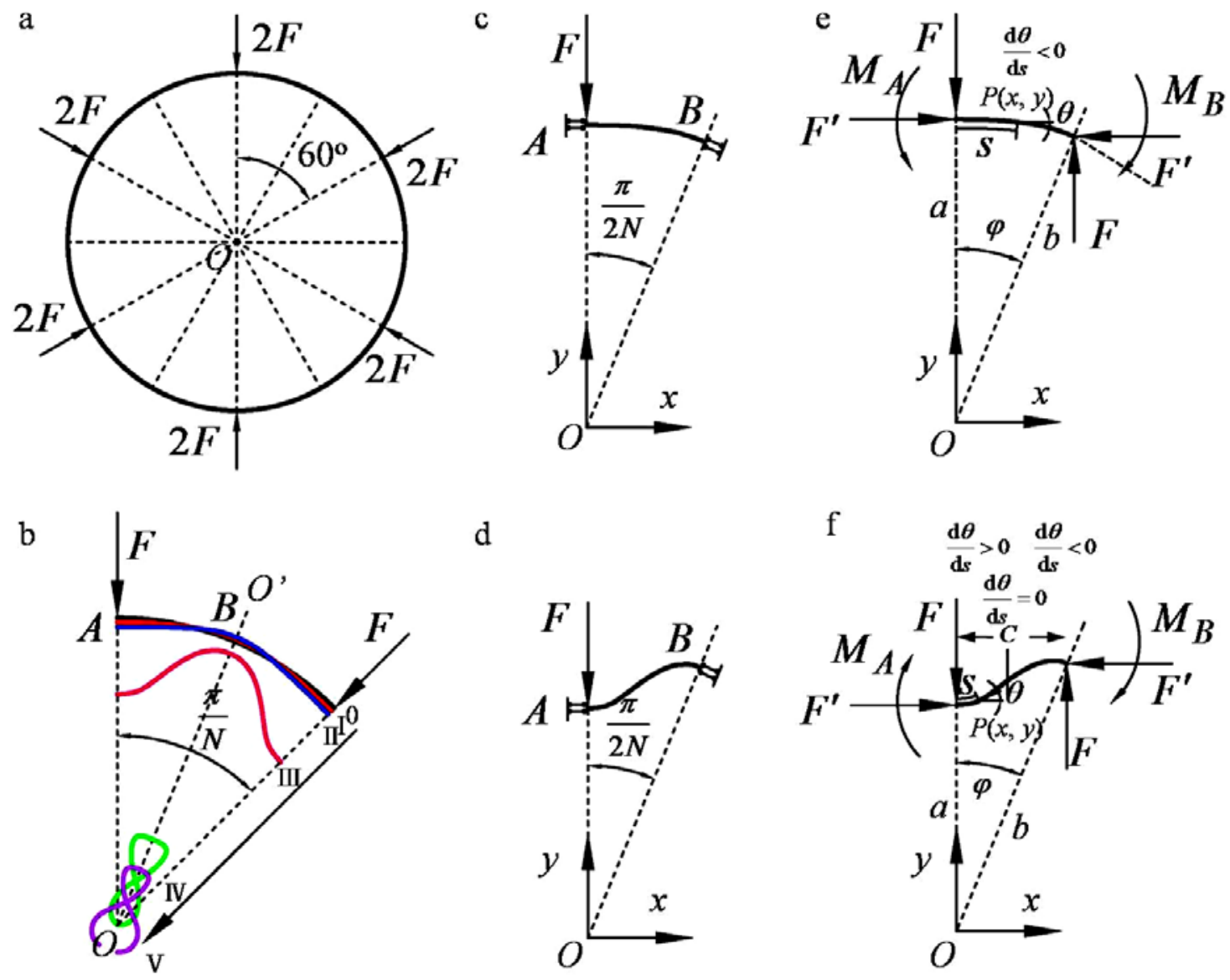

Figure 1. Schematics and force analysis of a ring with $N$-pairs of equal compressive radial forces.

(a) A 2D ring with three pairs of equal compressive radial forces; (b) $1 / 2 N$ ring with $N$-pairs of equal compressive forces, and six states with the increasing compressive forces; (c) half structure of the group 1; (d) half structure of the group 2; (e) force analysis of the group 1; (f) force analysis of the group 2. (The color is only available on web version) 


\subsection{Multi-pairs of equal radial compressive forces}

Here, we exemplify a 2D ring structure with three pairs of evenly distributed equal compressive forces as shown in figure 1a. From figure 1a, it is easily generalized that for the ring under $N$ pairs of evenly distributed equal forces, only a $1 / 2 N$ portion of the ring needs to be studied. Moreover, as the compressive forces increase, the ring experiences six states from 0 to $\mathrm{V}$ as marked in figure $1 \mathrm{~b}$. State 0 is an initial ring subjected to no force. The other five states, can be divided into two groups. Group 1 (comprising states I, II) is with negative curvature and group 2 (with states II - V) with both positive and negative curvatures. Apparently, the state II which is common to both groups is critical. Then, basing on the group division and considering only radial displacements at points $A$ and $B$, the half structures of the two groups are simplified as shown in figures 1c,d, and the forces analyzed are shown in figures 1e,f.

\subsubsection{Group 1 (States I, II)}

(a) State I (Red profile in figure 1b)

From figure 1e, under a vertical external force $F$ at the end point $A$, the vertical force at the end point $B$ equals $F$ because of the equilibrium in the vertical direction. Moreover, due to the guided constraint, the resultant force (or constraint reaction) of the horizontal force $F^{\prime}$ and the vertical force $F$ at the end point $B$ must be tangential to deformed rings at the point, thus, the horizontal force $F^{\prime}$ is calculated by $F^{\prime}=F \cot \varphi$ with $\varphi=\pi / 2 N$. Applying moment equilibrium with respect to the end point $A$, we obtain:

$$
-M_{A}+M_{B}=F b \xi(a, b) \text { with } \xi(a, b)=\frac{1-(a / b) \cos \varphi}{\sin \varphi}
$$

where $M_{A}$ and $M_{B}$ are moments acting at the end points $A$ and $B$, respectively, $a$ and $b$ are distances from the end points $A$ and $B$ to the origin $O$, i.e., ring center, respectively. Thus, 
employing the elastica theory, the moment-curvature relationship of an arbitrary point $P(x, y)$ is expressed as [22],

$$
D \frac{\mathrm{d} \theta}{\mathrm{d} s}=-\left[M_{B}-F(b \sin \varphi-x)+F^{\prime}(y-b \cos \varphi)\right]
$$

where $D$ is the bending rigidity of the ring, for bars $D=E I$ and for thin plates $D=E t^{2} / 12\left(1-v^{2}\right)$, (s, $\theta$ ) is the curvilinear coordinate of the point $P$. Here, $\theta$ is defined to be positive when the tangent line of the point $P$ pointing at the end point $B$ is counterclockwise relative to the positive $x$ axis, otherwise, it is negative, thus, when the point $P$ moves from $A$ to $B, \theta$ monotonously varies from 0 to $-\varphi$. Deriving Eq. (2) with respect to the curvilinear coordinate $s$ and considering the geometrical relationship $\mathrm{d} x / \mathrm{d} s=\cos \theta$ and $\mathrm{d} y / \mathrm{d} s=\sin \theta$, it is rewritten as,

$$
D \frac{\mathrm{d}^{2} \theta}{\mathrm{d} s^{2}}=-F \cos \theta-F^{\prime} \sin \theta
$$

multiplying both sides of Eq. (3) by $\mathrm{d} \theta / \mathrm{d} s$, and integrating it by substituting $F^{\prime}=F \cot \varphi$, its solution is obtained as,

$$
\frac{1}{2} D\left(\frac{\mathrm{d} \theta}{\mathrm{d} s}\right)^{2}=F \frac{\cos (\theta+\varphi)}{\sin \varphi}+C
$$

Boundary conditions provide $\mathrm{d} \theta /\left.\mathrm{d} s\right|_{\theta=0}=-M_{A} / D$ and $\mathrm{d} \theta /\left.\mathrm{d} s\right|_{\theta=-\varphi}=-M_{B} / D$, which lead to two expressions with respect to $M_{A}$ and $M_{B}$. Invoking Eq. (1) and solving the two expressions, we obtain another equation:

$$
M_{A}+M_{B}=\frac{2 D}{b} \zeta(a, b) \text { with } \zeta(a, b)=\frac{1-\cos \varphi}{1-(a / b) \cos \varphi}
$$

Then, combining Eqs. (1), (4) and (5), the unknowns $M_{A}, M_{B}$ and $C$ are calculated as:

$$
\left\{\begin{array}{l}
M_{A}=-\frac{F b}{2} \xi(a, b)+\frac{D}{b} \zeta(a, b) \\
M_{B}=\frac{F b}{2} \xi(a, b)+\frac{D}{b} \zeta(a, b) \\
C=\frac{M_{A}^{2}}{2 D}-\frac{F \cos \varphi}{\sin \varphi} \text { or } \frac{M_{B}^{2}}{2 D}-\frac{F}{\sin \varphi}
\end{array}\right.
$$

The length of the $\operatorname{arc} A B$ is $l=\varphi R$, and defining a set of non-dimensional quantities, $k^{2}=F l^{2} / D$, 
$\bar{a}=a / l, \bar{b}=b / l, \bar{s}=s / l$, the non-dimensional curvature is solved from Eq. (4) as:

$$
\frac{\mathrm{d} \theta}{\mathrm{d} \bar{s}}=-\sqrt{\frac{2}{\sin \varphi}} k \sqrt{K^{2}+\cos (\theta+\varphi)} \text { with } K^{2}=\frac{\sin \varphi}{2 k^{2} \bar{b}^{2}}\left(\frac{k^{2} \bar{b}^{2}}{2} \xi(\bar{a}, \bar{b})-\zeta(\bar{a}, \bar{b})\right)^{2}-\cos \varphi
$$

here, since $\theta$ monotonously decreases from 0 to $-\varphi, \mathrm{d} \theta / \mathrm{d} \bar{s}$ is negative, and the curvilinear coordinate of the arbitrary point $P$ is integrated as,

$$
\bar{s}(\theta)=\sqrt{\sin \varphi} \frac{p}{k}\left[F\left(\frac{\varphi}{2}, p\right)-F\left(\frac{\varphi}{2}+\frac{\theta}{2}, p\right)\right]
$$

where $p=\sqrt{2 /\left(1+K^{2}\right)}$, and $F(\psi, p)=\int_{0}^{\psi} \mathrm{d} \psi / \sqrt{1-p^{2} \sin ^{2} \psi}$ is the incomplete elliptical integral of the first kind, in which $\psi$ represents a general amplitude. Due to the neglect of axial and shear deformations, the inextensible condition $\bar{s}(-\varphi)=1$ holds, and the following equation arrives:

$$
\sqrt{\sin \varphi} \frac{p}{k} F\left(\frac{\varphi}{2}, p\right)=1
$$

To determine the deformation of the structures, the Cartesian coordinates of the point $P$ are always calculated by integrating the vector $\operatorname{dr}(\theta)=\mathrm{d}(\bar{x}, \bar{y})^{T}=(\cos \theta, \sin \theta)^{T} \mathrm{~d} \bar{s}$, where $\bar{x}=x / l$ and $\bar{y}=y / l$, i.e.:

$$
r(\theta)=-\frac{1}{k} \sqrt{\frac{\sin \varphi}{2}}\left(\int_{0}^{\theta} \frac{\cos \theta}{\sqrt{K^{2}+\cos (\theta+\varphi)}} \mathrm{d} \theta, \int_{0}^{\theta} \frac{\sin \theta}{\sqrt{K^{2}+\cos (\theta+\varphi)}} \mathrm{d} \theta\right)^{T}+(0, \bar{a})^{T}
$$

furthermore, equation (10) is solved as (See the derivation in appendix A):

$$
\boldsymbol{r}(\theta)=-\frac{1}{k} \sqrt{\frac{\sin \varphi}{2}}\left[\begin{array}{cc}
\sin \varphi & \cos \varphi \\
\cos \varphi & -\sin \varphi
\end{array}\right]\left(\begin{array}{c}
A(\theta) \\
B(\theta)
\end{array}\right)+\left(\begin{array}{c}
0 \\
\bar{a}
\end{array}\right)
$$

where

$$
\left\{\begin{array}{l}
A(\theta)=-2\left[\sqrt{K^{2}+\cos (\theta+\varphi)}-\sqrt{K^{2}+\cos \varphi}\right] \\
B(\theta)=-\sqrt{2} p\left(K^{2}\left[F\left(\frac{\theta}{2}+\frac{\varphi}{2}, p\right)-F\left(\frac{\varphi}{2}, p\right)\right]-\frac{2}{p^{2}}\left[E\left(\frac{\theta}{2}+\frac{\varphi}{2}, p\right)-E\left(\frac{\varphi}{2}, p\right)\right]\right)
\end{array}\right.
$$

in which $E(\psi, p)=\int_{0}^{\psi} \sqrt{1-p^{2} \sin ^{2} \psi} \mathrm{d} \psi$ is the incomplete elliptical integral of the second kind, $\psi$ also represents a general amplitude. It is readily seen that Eqs. (11) and (12) give $r(0)=(0, \bar{a})^{T}$, which is the coordinate of the end point $A$. For the end point $B$, equation (11) should satisfy 
$\boldsymbol{r}(-\varphi)=\bar{b}(\sin \varphi, \cos \varphi)^{T}$, which are expressed as,

$$
\left\{\begin{array}{l}
\bar{x}(-\varphi)=-\frac{1}{k} \sqrt{\frac{\sin \varphi}{2}}[A(-\varphi) \sin \varphi+B(-\varphi) \cos \varphi]=\bar{b} \sin \varphi \\
\bar{y}(-\varphi)=-\frac{1}{k} \sqrt{\frac{\sin \varphi}{2}}[A(-\varphi) \cos \varphi-B(-\varphi) \sin \varphi]+\bar{a}=\bar{b} \cos \varphi
\end{array}\right.
$$

Addressing Eq. (13), on the one hand, we multiply the first equation by $\sin \varphi$, the second by $\cos \varphi$, and sum them together; on the other hand, we multiply the first equation by $\cos \varphi$, the second by $\sin \varphi$, and subtract the first by the second, then, we obtain:

$$
\left\{\begin{array}{l}
-\frac{1}{k} \sqrt{\frac{\sin \varphi}{2}} A(-\varphi)=\bar{b}-\bar{a} \cos \varphi \\
-\frac{1}{k} \sqrt{\frac{\sin \varphi}{2}} B(-\varphi)=\bar{a} \sin \varphi
\end{array}\right.
$$

It is noted that the first expression in Eq. (14) holds (See the proof in appendix B). Replacing $\theta$ in $B(\theta)$ of Eq. (12) by $-\varphi$ and using the inextensible condition Eq. (9), the second expression of Eq. (14) is re-expressed as:

$$
\bar{a} \sin \varphi+K^{2}-\frac{2 \sqrt{\sin \varphi}}{k p} E\left(\frac{\varphi}{2}, p\right)=0
$$

According to Eqs. (9) and (15), the shape parameters $\bar{a}$ and $\bar{b}$ reflecting the deformation of the ring, are implicit functions of the force $k$, which determines the profile of the ring.

Moreover, the elastic strain energy is derived as:

$$
U_{\mathrm{I}}=\int_{0}^{l} \frac{1}{2} D\left(\left|\frac{\mathrm{d} \theta}{\mathrm{d} s}\right|-\kappa_{0}\right)^{2} \mathrm{~d} s
$$

where $\kappa_{0}=1 / R=\varphi / l$ is the initial curvature (i.e. curvature of the state 0 ). Re-expressing it in a non-dimensional form and employing Eq. (7), we find:

$$
\bar{U}_{\mathrm{I}}=\frac{U_{\mathrm{I}} l}{D}=\frac{1}{2} \int_{0}^{1}\left(\left|\frac{\mathrm{d} \theta}{\mathrm{d} \bar{s}}\right|-\kappa_{0} l\right)^{2} \mathrm{~d} \bar{s}=\frac{2}{\sqrt{\sin \varphi}} \frac{k}{p} E\left(\frac{\varphi}{2}, p\right)-\frac{\varphi^{2}}{2}
$$

(b) State II (Blue profile in figure 1b)

Regarding the critical state II transiting from the states I and III in figure $1 \mathrm{~b}$, it is a particular case common to both states. In this state, the curvature at the end point $A$ starts changing from 
negative to positive (figure 1e,f), i.e., $\mathrm{d} \theta /\left.\mathrm{d} \bar{s}\right|_{\theta=0}=0$. Employing Eq. (7), the zero curvature provides $K^{2}=-\cos \varphi$, which further leads to $p=\csc (\varphi / 2)$ by $p=\sqrt{2 /\left(1+K^{2}\right)}$. Then, substituting $p=\csc (\varphi / 2)$ into the inextensible condition (9), the critical force $k_{c r, 1}$ is expressed as,

$$
k_{c r, 1}=\sqrt{2 \cot \left(\frac{\varphi}{2}\right)} F\left(\frac{\varphi}{2}, \csc \frac{\varphi}{2}\right)
$$

and inserting $K^{2}=-\cos \varphi, \quad p=\csc (\varphi / 2)$ and $k_{c r, 1}$ into Eq. (15), the shape parameter $\bar{a}_{c r, 1}$ is calculated as:

$$
\bar{a}_{c r, 1}=\csc \varphi\left[\cos \varphi+(1-\cos \varphi) \frac{E\left(\frac{\varphi}{2}, \csc \frac{\varphi}{2}\right)}{F\left(\frac{\varphi}{2}, \csc \frac{\varphi}{2}\right)}\right]
$$

moreover, $K^{2}=-\cos \varphi$ also leads to the following expression by the definition of $K^{2}$ in Eq. (7):

$$
k^{2} \bar{b}^{2}=2 \frac{\zeta(\bar{a}, \bar{b})}{\xi(\bar{a}, \bar{b})}
$$

and inserting $k_{c r, 1}$ and $\bar{a}_{c r, 1}$ into Eq. (20), $\bar{b}_{c r, 1}$ is calculated as:

$$
\bar{b}_{c r, 1}=\cot \varphi \cos \varphi+(1-\cos \varphi) \frac{1+\cot \varphi \cdot E\left(\frac{\varphi}{2}, \csc \frac{\varphi}{2}\right)}{F\left(\frac{\varphi}{2}, \csc \frac{\varphi}{2}\right)}
$$

\subsubsection{Group 2 (States II, III, IV, and V)}

State II is the critical state between groups 1 and 2, and it has already been dealt within Section 2.1.1. (b), thus, it is not intended to be derived in this Section.

(c) State III (Plum profile in figure 1b)

As stated before, differently from the state I, the positive and negative curvatures exist simultaneously as the force increases, and there is an inflection point $C$, see figure $1 \mathrm{f}$. For the $A C$ part, $\mathrm{d} \theta / \mathrm{d} \bar{s}>0$, and for the $C B$ part, $\mathrm{d} \theta / \mathrm{d} \bar{s}<0$, thus, at the inflection point $C, \mathrm{~d} \theta /\left.\mathrm{d} \bar{s}\right|_{\theta_{c r}}=0$, where $\theta_{c r}$ is the angular coordinate of the point $C$. Here we mark the two parts $A C$ and $C B$ by subscripts 1 and 2 respectively, and the curvilinear coordinates of the point $P$ in the two parts are 
calculated by integrating the following curvature expressions:

$$
\begin{cases}\frac{\mathrm{d} \theta}{\mathrm{d} \bar{s}_{1}}=\sqrt{\frac{2}{\sin \varphi} k \sqrt{K^{2}+\cos (\theta+\varphi)}} & \theta \in\left(0, \theta_{c r}\right) \\ \frac{\mathrm{d} \theta}{\mathrm{d} \bar{s}_{2}}=-\sqrt{\frac{2}{\sin \varphi} k \sqrt{K^{2}+\cos (\theta+\varphi)}} & \theta \in\left(\theta_{c r},-\varphi\right)\end{cases}
$$

where $K^{2}$ is the same as that in Eq. (7), and Eq. (22) is integrated as:

$$
\begin{cases}\bar{s}_{1}(\theta)=\sqrt{\sin \varphi} \frac{p}{k}\left[F\left(\frac{\varphi}{2}+\frac{\theta}{2}, p\right)-F\left(\frac{\varphi}{2}, p\right)\right] & \theta \in\left(0, \theta_{c r}\right) \\ \bar{s}_{2}(\theta)=\sqrt{\sin \varphi} \frac{p}{k}\left[F\left(\frac{\varphi}{2}+\frac{\theta_{c r}}{2}, p\right)-F\left(\frac{\varphi}{2}+\frac{\theta}{2}, p\right)\right] & \theta \in\left(\theta_{c r},-\varphi\right)\end{cases}
$$

Then, the inextensible condition provides $\bar{s}_{1}\left(\theta_{c r}\right)+\bar{s}_{2}(-\varphi)=1$, which is further expressed as:

$$
\sqrt{\sin \varphi} \frac{p}{k}\left[2 F\left(\frac{\varphi}{2}+\frac{\theta_{c r}}{2}, p\right)-F\left(\frac{\varphi}{2}, p\right)\right]=1
$$

Similar as Eqs. (10-12), the Cartesian coordinate of the point $P$ in the part $A C$ is computed as:

$$
r_{1}(\theta)=\frac{1}{k} \sqrt{\frac{\sin \varphi}{2}}\left[\begin{array}{cc}
\sin \varphi & \cos \varphi \\
\cos \varphi & -\sin \varphi
\end{array}\right]\left(\begin{array}{c}
A_{1}(\theta) \\
B_{1}(\theta)
\end{array}\right)+\left(\begin{array}{c}
0 \\
\bar{a}
\end{array}\right)
$$

where $A_{1}(\theta)$ and $B_{1}(\theta)$ are same as Eq. (12), but the upper limit of the angular coordinate $\theta$ is replaced by $\theta_{c r}$ instead of $-\varphi$. Equation (25) also provides the coordinates of the end point $A$, i.e., $\boldsymbol{r}_{1}(0)=(0, \bar{a})^{T}$. For the part $C B$, the Cartesian coordinate is calculated as:

$$
r_{2}(\theta)=-\frac{1}{k} \sqrt{\frac{\sin \varphi}{2}}\left[\begin{array}{cc}
\sin \varphi & \cos \varphi \\
\cos \varphi & -\sin \varphi
\end{array}\right]\left(\begin{array}{l}
A_{2}(\theta) \\
B_{2}(\theta)
\end{array}\right)+r_{1}\left(\theta_{c r}\right)
$$

where

$$
\left\{\begin{array}{l}
A_{2}(\theta)=-2 \sqrt{K^{2}+\cos (\theta+\varphi)} \\
B_{2}(\theta)=-\sqrt{2} p\left(K^{2}\left[F\left(\frac{\theta}{2}+\frac{\varphi}{2}, p\right)-F\left(\frac{\theta_{c r}}{2}+\frac{\varphi}{2}, p\right)\right]-\frac{2}{p^{2}}\left[E\left(\frac{\theta}{2}+\frac{\varphi}{2}, p\right)-E\left(\frac{\theta_{c r}}{2}+\frac{\varphi}{2}, p\right)\right]\right)
\end{array}\right.
$$

As stated above, at the inflection point $C, \mathrm{~d} \theta /\left.\mathrm{d} \bar{s}\right|_{\theta_{c r}}=0$ holds, which leads to $K^{2}+\cos \left(\theta_{c r}+\varphi\right)=0$ from Eq. (22) and $A_{2}\left(\theta_{c r}\right)=B_{2}\left(\theta_{c r}\right)=0$ from Eq. (27), thus, equation (26) provides $\boldsymbol{r}_{2}\left(\theta_{\boldsymbol{c}}\right)=\boldsymbol{r}_{1}\left(\theta_{\boldsymbol{c}}\right)$, which ensures the continuity of the parts $A C$ and $C B$ at the point $C$. Moreover, using Eq. (26) and considering $\boldsymbol{r}_{2}(-\varphi)=\bar{b}(\sin \varphi, \cos \varphi)^{T}$, then like with treatment of Eq. (13), we obtained: 


$$
\left\{\begin{array}{l}
\frac{1}{k} \sqrt{\frac{\sin \varphi}{2}}\left[A_{1}\left(\theta_{c r}\right)-A_{2}(-\varphi)\right]+\bar{a} \cos \varphi=\bar{b} \\
\frac{1}{k} \sqrt{\frac{\sin \varphi}{2}}\left[B_{1}\left(\theta_{c r}\right)-B_{2}(-\varphi)\right]-\bar{a} \cos \varphi=0
\end{array}\right.
$$

As in the proof in appendix B, the first equation in Eq. (28) always holds, and the second expression yields:

$$
\bar{a} \sin \varphi+K^{2}-\frac{2 \sqrt{\sin \varphi}}{k p}\left[2 E\left(\frac{\varphi}{2}+\frac{\theta_{c r}}{2}, p\right)-E\left(\frac{\varphi}{2}, p\right)\right]=0
$$

employing Eqs. (24) and (29), the shape parameters $\bar{a}$ and $\bar{b}$ can be solved by a given force $k$.

For the state III, the elastic strain energy is calculated in a non-dimensional form as:

$$
\bar{U}_{\mathrm{III}}=\frac{U_{\mathrm{III}} l}{D}=\frac{1}{2} \int_{0}^{1}\left(\left|\frac{\mathrm{d} \theta}{\mathrm{d} \bar{s}}\right|-\kappa_{0} l\right)^{2} \mathrm{~d} \bar{s}=\frac{2}{\sqrt{\sin \varphi}} \frac{k}{p}\left[2 E\left(\frac{\theta_{c r}+\varphi}{2}, p\right)-E\left(\frac{\varphi}{2}, p\right)\right]-2 \varphi \theta_{c r}-\frac{\varphi^{2}}{2}
$$

(d) State IV (Green profile in figure 1b)

State IV, in figure 1b, is the second critical state being between the states III and V. In this case, $\bar{a}_{c r, 2}=0$. Employing the inextensible condition Eqs. (24) and (29), the force $k_{c r, 2}$ and shape parameter $\bar{b}_{c r, 2}$ can be obtained.

(e) State V (Purple profile in figure 1b)

The calculation of state V is same as state III, so we here will not repeat the process.

From analyzing states I to $\mathrm{V}$, we can conclude that when the force $k$ varies from 0 to $k_{c r, 1}$, state I exists, when $k$ varies from $k_{c r, 1}$ to $k_{c r, 2}$, state III exists and state $\mathrm{V}$ emerges when $k$ is beyond $k_{c r, 2}$.

\subsection{Multi-pairs of equal tensile forces}

Section 2.1 deals with the five compressive states of the ring. For the tensile case, its curvature is similar to that of the state I in the compressive case, i.e., $\mathrm{d} \theta / \mathrm{d} \bar{s}<0$, where $\theta$ changes from 0 to $-\varphi$, and what we need do is to replace the force $F$ in Eqs. (4) and (6) with $-F$. Then, the curvature of the arbitrary point $P$ is expressed as: 


$$
\frac{\mathrm{d} \theta}{\mathrm{d} \bar{s}}=-\sqrt{\frac{2}{\sin \varphi}} k \sqrt{K^{2}-\cos (\theta+\varphi)} \text { with } K^{2}=\frac{\sin \varphi}{2 k^{2} \bar{b}^{2}}\left(\frac{k^{2} \bar{b}^{2}}{2} \xi(\bar{a}, \bar{b})+\zeta(\bar{a}, \bar{b})\right)^{2}+\cos \varphi
$$

and the curvilinear coordination is solved as:

$$
\bar{s}(\theta)=\sqrt{\sin \varphi} \frac{p}{k}\left[F\left(\frac{\pi+\varphi}{2}, p\right)-F\left(\frac{\pi+\varphi}{2}+\frac{\theta}{2}, p\right)\right] \text { with } K^{2}=\frac{\sin \varphi}{2 k^{2} \bar{b}^{2}}\left(\frac{k^{2} \bar{b}^{2}}{2} \xi(\bar{a}, \bar{b})+\zeta(\bar{a}, \bar{b})\right)^{2}+\cos \varphi
$$

where $p=\sqrt{2 /\left(1+K^{2}\right)}$. Invoking the inextensible condition $\bar{s}(-\varphi)=1$, we find:

$$
\sqrt{\sin \varphi} \frac{p}{k}\left[F\left(\frac{\pi+\varphi}{2}, p\right)-F\left(\frac{\pi}{2}, p\right)\right]=1
$$

Similar to the Cartesian expressions (11) and (25) of the point $P$, its vector is calculated as:

$$
\overrightarrow{\boldsymbol{r}}(\theta)=-\frac{1}{k} \sqrt{\frac{\sin \varphi}{2}}\left[\begin{array}{cc}
\sin \varphi & \cos \varphi \\
\cos \varphi & -\sin \varphi
\end{array}\right]\left(\begin{array}{c}
A(\theta) \\
B(\theta)
\end{array}\right)+\left(\begin{array}{c}
0 \\
\bar{a}
\end{array}\right)
$$

where

$$
\left\{\begin{array}{l}
A(\theta)=2\left[\sqrt{K^{2}-\cos (\theta+\varphi)}-\sqrt{K^{2}-\cos \varphi}\right] \\
B(\theta)=\sqrt{2} p\left(K^{2}\left[F\left(\frac{\theta}{2}+\frac{\pi+\varphi}{2}, p\right)-F\left(\frac{\pi+\varphi}{2}, p\right)\right]-\frac{2}{p^{2}}\left[E\left(\frac{\theta}{2}+\frac{\pi+\varphi}{2}, p\right)-E\left(\frac{\pi+\varphi}{2}, p\right)\right]\right)
\end{array}\right.
$$

considering the coordinates of the end point $B \quad r(-\varphi)=\bar{b}(\sin \varphi, \cos \varphi)^{T}$ and substituting it into Eq.

(34), like the treatment of Eq. (13), we obtained the following expression:

$$
\left\{\begin{array}{l}
-\frac{1}{k} \sqrt{\frac{\sin \varphi}{2}} A(-\varphi)=\bar{b}-\bar{a} \cos \varphi \\
-\frac{1}{k} \sqrt{\frac{\sin \varphi}{2}} B(-\varphi)=\bar{a} \sin \varphi
\end{array}\right.
$$

Similarly, the first equation in Eq. (37) also always holds, and the second expression yields:

$$
\bar{a} \sin \varphi-K^{2}+\frac{2 \sqrt{\sin \varphi}}{k p}\left[E\left(\frac{\pi+\varphi}{2}, p\right)-E\left(\frac{\pi}{2}, p\right)\right]=0
$$

Combing Eqs. (33) and (37), the shape parameters $\bar{a}$ and $\bar{b}$ can be solved by a given $k$.

Moreover, the non-dimensional elastic strain energy of the tensile case is calculated as,

$$
\bar{U}_{T}=\frac{U_{T} l}{D}=\frac{1}{2} \int_{0}^{1}\left(\left|\frac{\mathrm{d} \theta}{\mathrm{d} \bar{s}}\right|-\kappa_{0} l\right)^{2} \mathrm{~d} \bar{s}=\frac{2}{\sqrt{\sin \varphi}} \frac{k}{p}\left[E\left(\frac{\pi+\varphi}{2}, p\right)-E\left(\frac{\pi}{2}, p\right)\right]-\frac{\varphi^{2}}{2}
$$




\section{RESULTS AND DISCUSSIONS}

Here, we consider four loading cases, i.e., $N=1,2,3$, 4. For each case, one tensile state and six compressive states are theoretically treated, and the specific non-dimensional forces $k$ for the seven states are set to be $-6,0,0.8 k_{c r, 1}, k_{c r, 1}, 0.8 k_{c r, 2}, k_{c r, 2}, 2 k_{c r, 2}$. Correspondingly, the commercial software ABAQUS/Standard was used to verify the theoretical results. The radius of the ring in the FE geometrical model is $10 \mathrm{~mm}$, and the cross-section of the ring bar is circular, with the radius set to $0.1 \mathrm{~mm}$. The linear-elastic constitutive behavior of the ring material is used, and its Young's modulus and Poisson's ratio are $210 \mathrm{GPa}$ and 0.25 , respectively. Thanks to symmetry it the FE model was simplified, by studying a $1 / 2 N$ ring, see figure 2 . The model contains 100 2-node B21 elements which include the shear effect, and 101 uniformly distributed nodes. The loading process is displacement controlled. Since the analysis is a quasi-static large deformation, an automatic incrementing type is used to ensure the convergence. As for the continuous deformation process of the whole ring, it can be seen in supplementary video materials. 

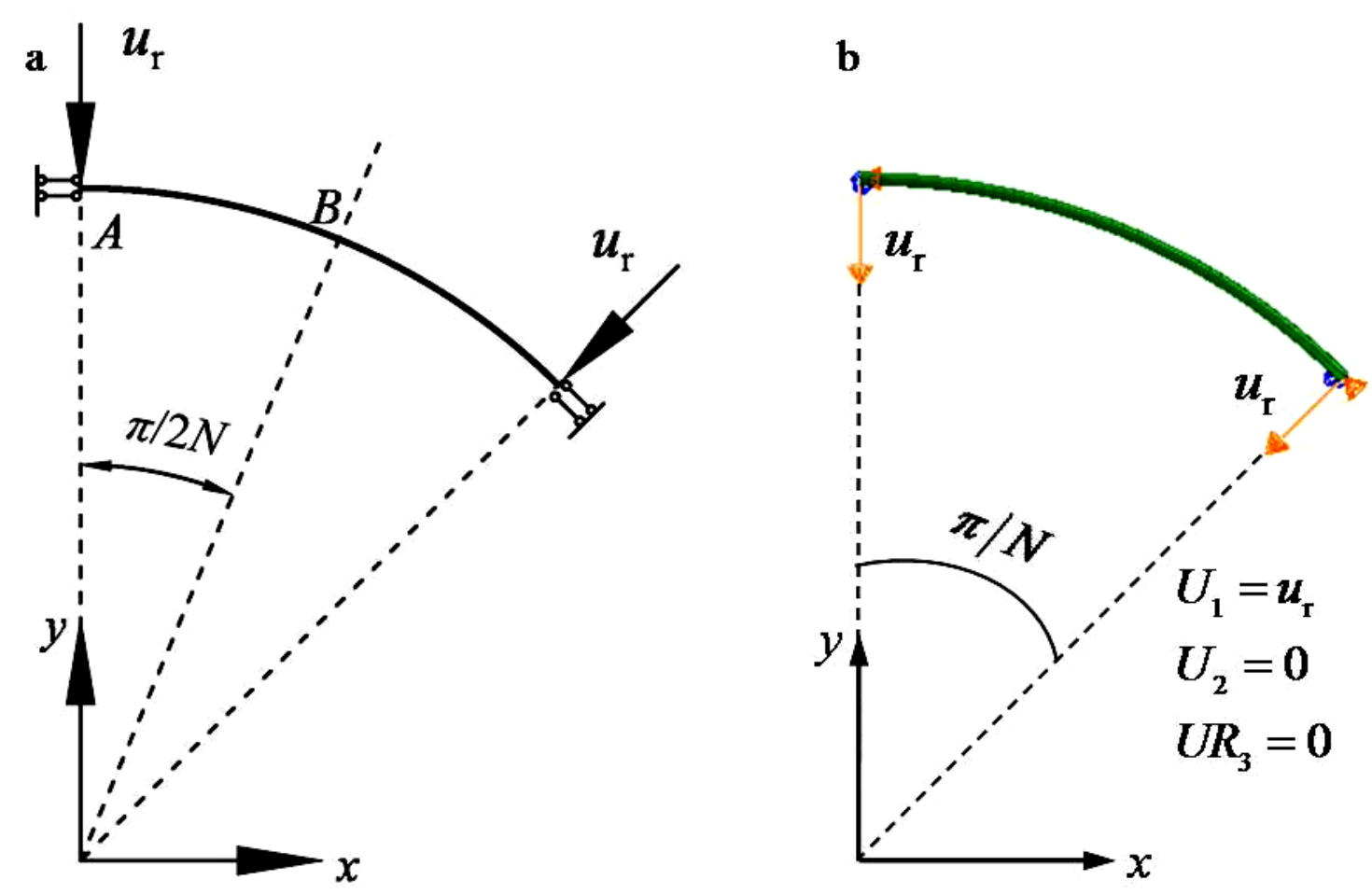

Figure 2. (a) Diagram and (b) mesh and boundary condition of finite element model. Note: $U_{1}$

and $U_{2}$ are the displacements in the radial and circumferential directions, respectively, and $U R_{3}$ is the rotation.

\subsection{Deformation Profiles}

Each of the seven specific states, is denoted by an orange, black, red, blue, plum, green, and purple profile respectively see figure 3 . The results show that the theoretical prediction and FE simulation agree very well (solid lines for theoretical results and the discrete markers for FE results in figure 3). It is easily seen that in the tensile state, the ring tends to be a regular polygon with $2 N$ sides as the force increases, for example, when $N=2,3,4$, the ring approaches square, regular hexagon and regular octagon (orange profiles in figures 3b-d), respectively. In the compressive states, the critical state II (blue profiles in figures 3a-d) gradually approaches the initial ring (black profiles in figures 3a-d) as $N$ increases, in particular, the critical state II in the case of $N=4$ almost coincides with the initial profile. That is to say, as $N$ increases, the scope of 
the state I (red profiles in figures 3a-d) between the state 0 and the critical state II, becomes narrower and narrower; instead, the state III (plum profiles in figures 3a-d) between the state II and the critical state IV (green profiles in figures 3a-d), gets wider and wider. Moreover, if the force exceeds the first critical value, i.e., $0.8 k_{c r, 2}, k_{c r, 2}$, the ring deforms into flower-like patterns, and, when the force increases beyond the second critical value, i.e., $2 k_{c r, 2}$, the ring reversely tends to be a regular polygon but with $2 N$ knots (purple profiles in figures 3a-d). In particular, when $N=1$, the ring deformed by $0.8 k_{c r, 2}$ (plum profiles in figure 3a) is similar to the dog-bone shaped single-wall carbon nanotube bundles, and when $N=3$, the ring deformed by -6 (orange line in figure 3c) resembles the hexagonal single-wall carbon nanotube bundles [23, 24]. In this regard, due to their similar cross-sectional shapes, the elastic strain energy of carbon nanotubes could be solved by the present method. However, for nanomaterials, we have to say that the theory based on elastica theory is continuous and care should be exercised when dealing with discrete nanomaterials, e.g. nanotubes. In addition, when structural size enters the nano-scale, surface effects have to be taken into account [25]. 

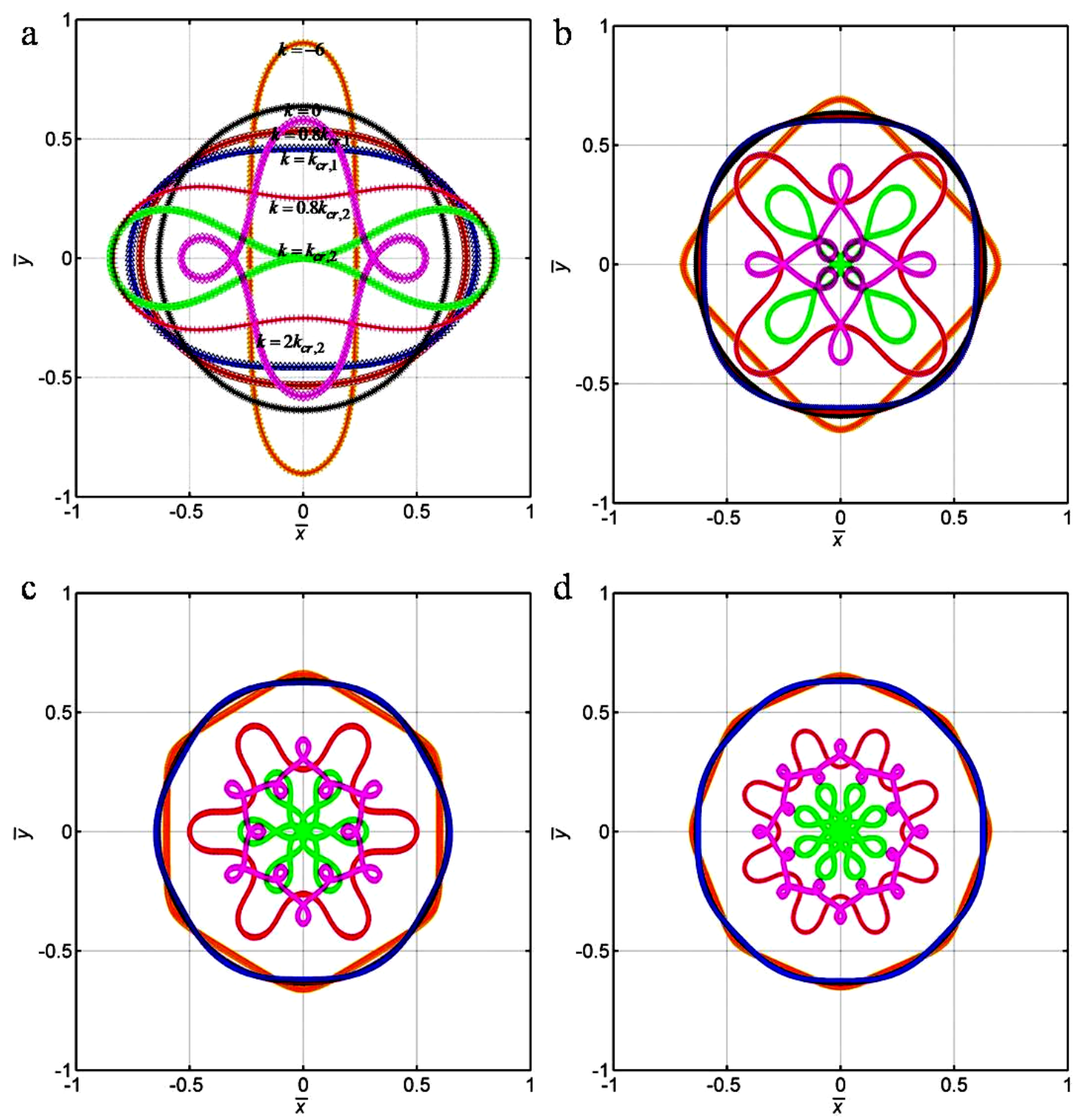

Figure 3. Comparison between theoretical and finite element results under $N$-pairs of equal forces

for each state. (a) $N=1$; (b) $N=2$; (c) $N=3$; (d) $N=4$. Note, the solid profiles are theoretical results, and the discrete marker profiles are FE results, because of the tightness between state 0 , state I and state II, it is not easy to distinguish them in figures (c) and (d). (The color is only available on web version).

As stated at the beginning of the Section 2, the static deformation theory of a 2D elastica ring derived mathematically does not include the in-plane/out-of-plane stability. Here, in the FE model, 
the in-plane/out-of-plane stability is guaranteed to use the displacement loading mode [26] and the 2D beam element. It is noted that the displacement loading mode of the FE model is different from the present theory, which is based on the force loading mode. In reality, the two loading modes may result in different structural stabilities under the compressive loads, because the ring structures are always stable in the displacement loading manner due to the prescribed displacement, and often unstable in the force loading manner due to the critical buckling force. Moreover, it is not easy to arrive at the deformations of states I- V as the structural instability of the $2 \mathrm{D}$ ring structures is present during the compressive process. In particular, the states I - III are also valid for 3D ring structures, which due to their larger axial dimensions are more stable during the deformation process than the $2 \mathrm{D}$ ring. The out-of-plane instability of the $2 \mathrm{D}$ rings is mainly caused by two aspects. One is the out-of-plane deformation of the ring arch in figure $1 \mathrm{~b}$ under lateral loads [27] before self-contact, which may occur in the states I - III. The other is the out-of-plane kinematics after self-contact [28], which may occur in the states III - V, furthermore a knot will be formed as the force increases, and this physical phenomenon can be often found in the study of other thin elastic rods $[28,29]$. Thus, the states IV and V, only occur when the ring is self-intersected or out-of-plane kinematics are permitted, and the characterization of self-intersection and out-of-plane kinematics that are both physically reasonable and mathematically precise, is very difficult [29].

To illustrate the effectiveness of the present theory, topological experiments were carried out to compare the geometrical similarity using a Nitrile Butadiene Rubber (NBR) ring, whose characteristics allowed it to be controlled more easily when entering into unstable states. The NBR ring was constrained between a base and transparent cover to control its out-of-plane kinematics. 
The corresponding loading cases were performed by tensioning cotton threads previously glued to the relevant points on the ring. For the tensile case, the tension was applied outwards, but for the compressive case, the tension was applied inwards. It is worth mentioning that in spite of using NBR for the ring, it was still very difficult to create the state $V$ due to the complexity of the pattern and the instability in the ring. In any case, the experimental result was obtained by integrating the raw pictures in Appendix $C$ and reported in figure 4, which partially show the consistence of the present theory.
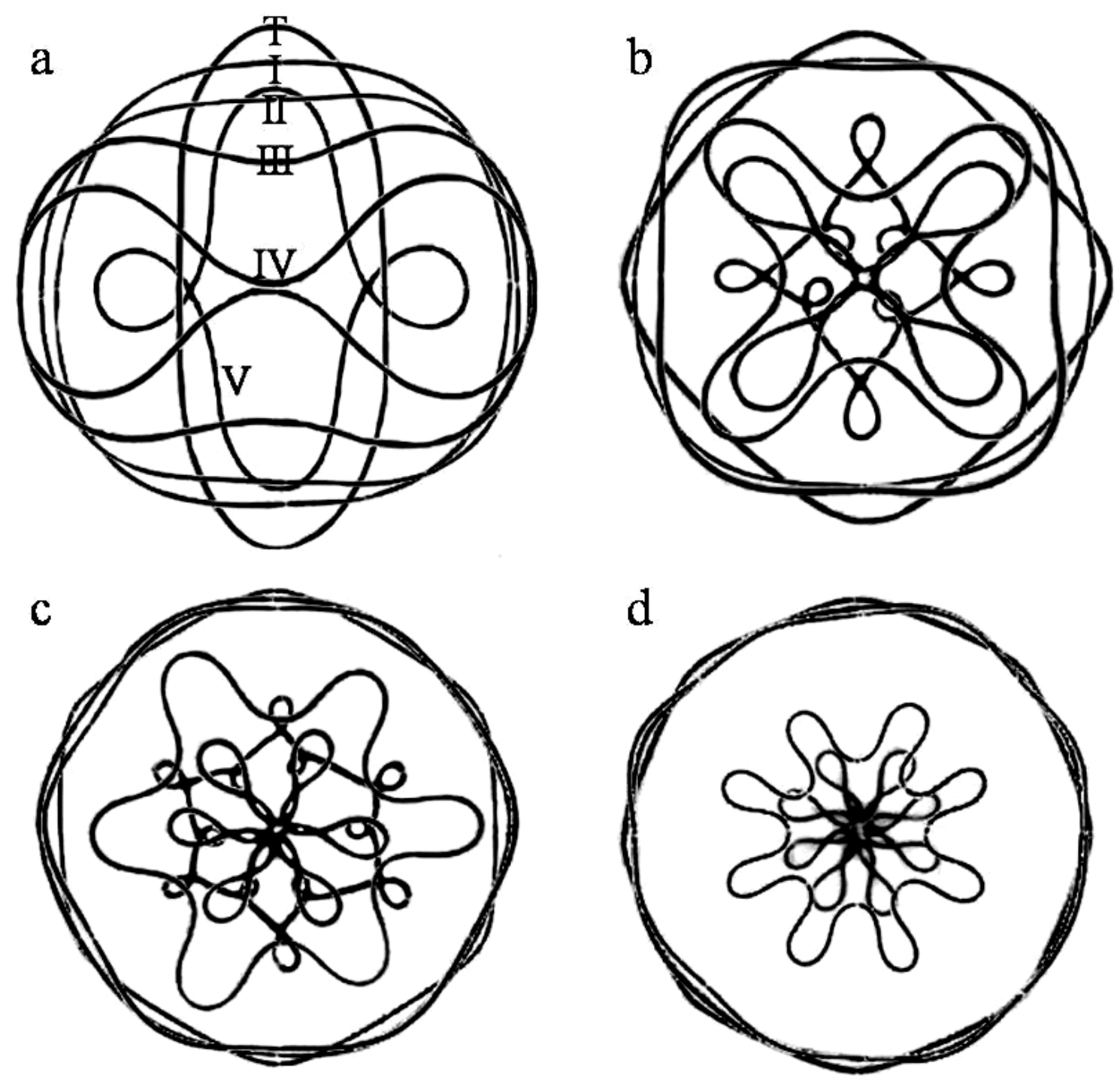

Figure 4. Topological experiments of extrinsic similar shapes as their counterparts in figure 3.

\subsection{Shape Parameters and Elastic Strain Energy}


For the loading case with $N$ pairs of forces, the force $F$, the shape parameters $a$ and $b$, energy $U$ are non-dimensionalized by $l=\varphi R=\frac{\pi}{2 N} R=\frac{L}{4 N}$, where $L=2 \pi R$ is the perimeter of the ring, and they are expressed as $k=\frac{L}{4 N} \sqrt{\frac{F}{D}}, \bar{a}=\frac{4 N a}{L}, \bar{b}=\frac{4 N b}{L}$, and $\bar{U}=\frac{U}{D} \cdot \frac{L}{4 N}$, respectively. Thus, because of the variable $N$ from 1 to 4 , the comparison of the shape parameters and elastic strain energy needs to be unified, and $N k=\frac{L}{4} \sqrt{\frac{F}{D}}, \frac{\bar{a}}{N}=\frac{4 a}{L}, \frac{\bar{b}}{N}=\frac{4 b}{L}$, and $N \bar{U}$ are here compared. Then, for the four above loading cases, the relationships of the shape parameters and elastic strain energies with forces are plotted in figure 5.
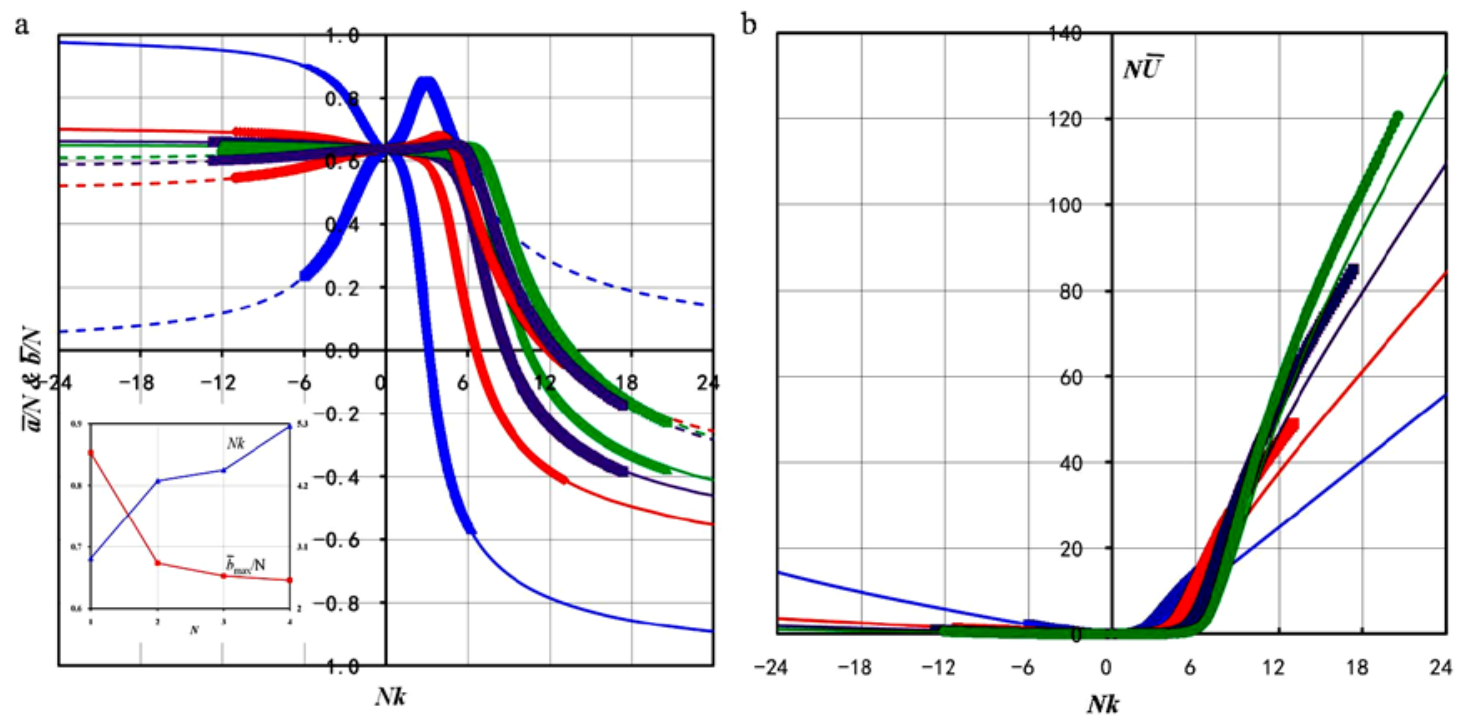

Figure 5. Shape parameters of loaded rings and strain energy stored in the $1 / 2 N$ rings. (a)

Variations of the shape parameters $\bar{a}$ and $\bar{b}$, for the theoretical results, the solid lines represent the shape parameter $\bar{a}$, and the dashed denote $\bar{b}$; (b) strain energy stored in $1 / 2 N$ ring. Noted that the blue, red, purple and green lines represent $N=1,2,3,4$ in order. (The color is only available on web version).

From figure 5a, it can be seen that the theoretical results (thin lines) match the finite element results (thick lines) very well. Regarding the scopes of the parameters $\bar{a}$ (solid lines in figure 5a) 
and $\bar{b}$ (dashed lines in figure 5a), we consider two extreme states, one is tensile and the other is compressive, and as stated before, the profiles of the ring in the two extreme states are approximate regular polygons, then, through the geometrical analysis on the polygons, the shape parameter $a$ varies from $-l \csc \varphi$ to $l \csc \varphi$, and $b$ from $-l \cot \varphi$ to $l \cot \varphi$. Thus, normalized by the arc length of $l$, the non-dimensional shape parameters $\bar{a}$ and $\bar{b}$ vary in the intervals of $(-\csc \varphi, \csc \varphi)$ and $(-\cot \varphi, \cot \varphi)$, respectively. In the tensile case, the non-dimensional force $k$ is negative. As the force increases, the shape parameters $\bar{a}$ and $\bar{b}$ increase and decrease, respectively, moreover, $\bar{a} / N$ tends to $\csc \varphi / N$, and $\bar{b} / N$ tends to $\cot \varphi / N$. For example, if there is one pair of equal tensile forces (blue lines in figure 5a), i.e., $N=1$ and $\varphi=\frac{\pi}{2}, \bar{a}$ tends to be 1.0 , and $\bar{b}$ to be 0 . This means that the $y$-coordinate of point $A$ approaches a quarter of the ring's perimeter, and point $B$ tends to the center of the ring. Plus, as $N$ increases, $\bar{a}$ and $\bar{b}$ tend to $\frac{2}{\pi}$. In the compressive case, the force $k$ is positive. As the force increases, $\bar{a}$ always decreases, but $\bar{b}$ increases first and then decreases, which can be clearly observed in figure 3a, i.e., in the case of $N=1, \bar{b}$ increases from state 0 (black profile) to state IV (green profile), and then decrease afterward (purple profile), similarly, $\bar{a} / N$ tends to $-\csc \varphi / N$, and $\bar{b} / N$ tend to $-\cot \varphi / N$. Meanwhile, as $N$ increases, the maximum of $\bar{b}$ and its corresponding $\bar{a}$ both tend to $\frac{2}{\pi}$ (see figure 5a and its inset), and its corresponding forces also increase (see the inset in figure 5a). This indicates that when $N$ tends to infinity, the ring's shape is still a circle even though a great compressive force is applied, and this can be used to explain a well-known phenomenon: when an egg is held in one hand, it does not break in spite that a large handgrip force is applied.

Figure $5 b$ depicts the elastic strain energy stored in the $1 / 2 N$ ring as the force increases. Even though the results from the two methods do not match very well as the profiles in figure 3 and 
shape parameters in figure 5a, they are still comparable. This is because the bending strain energy is only considered in the theory (thin lines), and compared to the bending deformation, the tensile and shear strain energies are neglected thanks to their smallness. However, the data from the finite element analysis (thick lines) includes all three, and thus the finite element result is a little greater than the theoretical results, and the difference grows as the external force increases. Plus, it shows that in the tensile case, the elastic strain energy is very small, and as $N$ increases, the elastic strain energy decreases. This is due to the inextensible condition which determines smaller and smaller deformation as $N$ increases. The compressive case has a very different behaviour to the tensile case, namely, as $N$ increases, the elastic strain energy increases, and this is due to a large number of applied forces on the ring with a greater deformation of the ring.

The present theoretical model for a ring structure is very useful for analyzing the mechanical behavior of micro- and nano-devices in MEMs, NEMs and electronics [30], however in these cases, distinct physical mechanisms, such as surface effect and size effect, in micro- or nano-scale should be further considered. In particular, for the stretchability and flexibility of electronics, the mechanical safety and electronic performance under large strain is not guaranteed [21], thus, the strain distribution of the micro-structure, particularly, the maximum strain is of much importance. It is critical to accurately predict the maximum strain using a theoretical model so that premature failure of the structure can be avoided by an optimal design. For the present ring structure, the well-known strain of the bending Euler beam along a cross-section is expressed as: $\varepsilon(y)=y \frac{\mathrm{d} \theta}{\mathrm{ds}}=\frac{M}{E I} y$ where $y$ is the distance from a material point to the neutral axis of the cross-section, thus with a uniform cross-section, the maximum strain depends on the curvature/moments and the cross-section size. From the Eq. (6), we can see that in the 
compressive case, $M_{\mathrm{B}}>M_{\mathrm{A}}$ always holds, and in the tensile case, $M_{\mathrm{B}}<M_{\mathrm{A}}$, so it is very easy to find the location of maximum strain, and so avoid the failure of the structure.

Finally, it is worth mentioning that care should be exercised when using this method for rings under unevenly distributed forces, which will induce non-symmetric configurations, and this is because the positions of the equivalent guided points $A$ and $B$ are not apparent as with the present work, and they must be determined through calculation.

\section{CONCLUSIONS}

In summary, we have developed a set of theories for a ring subjected to multi-pairs of evenly distributed equal radial forces. The theory reflects the relationship between the pair number of forces, the value of the forces, the shape and the strain energy. In the tensile case, there is only one state; while in the compressive case, the ring experiences six states. The results show a very good agreement between the theory and finite element results. Interestingly, no matter whether the ring is compressed or tensioned by $\mathrm{N}$-pairs of equal forces, it always tends to form a regular polygon with $2 N$ sides as the force increases, and with a compressive force the ring deforms into flower-like patterns. These results could be helpful in studying the mechanics of ring structures under lateral concentrated loads, such as the micro- or nano- devices in the MEM or NEM systems.

\section{Acknowledgements}

This work is partially supported by the National Natural Science Foundation of China (NSFC) (Nos. 31300780, 11272091, 11422222, 31470043), the Fundamental Research Funds for the 
Central Universities (No. 2242016R30014), and ARC (FT140101152). N.M.P. is supported by the European Research Council PoC 2015 "Silkene" No. 693670, by the European Commission H2020 under the Graphene Flagship Core 1 No. 696656 (WP14 "Polymer Nanocomposites") and under the FET Proactive "Neurofibres" No. 732344.

\section{Appendix A:}

The coordinate vector (10) of the point $P$ can be solved as:

$$
\begin{aligned}
& \vec{r}(\theta)=-\frac{1}{k} \sqrt{\frac{\sin \varphi}{2}}\left(\int_{0}^{\theta} \frac{\cos \theta}{\sqrt{K^{2}+\cos (\theta+\varphi)}} \mathrm{d} \theta, \int_{0}^{\theta} \frac{\sin \theta}{\sqrt{K^{2}+\cos (\theta+\varphi)}} \mathrm{d} \theta\right)^{T}+(0, \bar{a})^{T} \\
& =-\frac{1}{k} \sqrt{\frac{\sin \varphi}{2}}\left(\int_{0}^{\theta} \frac{\cos (\theta+\varphi-\varphi)}{\sqrt{K^{2}+\cos (\theta+\varphi)}} \mathrm{d} \theta, \int_{0}^{\theta} \frac{\sin (\theta+\varphi-\varphi)}{\sqrt{K^{2}+\cos (\theta+\varphi)}} \mathrm{d} \theta\right)^{T}+(0, \bar{a})^{T} \\
& =-\frac{1}{k} \sqrt{\frac{\sin \varphi}{2}}\left[\left(\sin \varphi \int_{0}^{\theta} \frac{\sin (\theta+\varphi)}{\sqrt{K^{2}+\cos (\theta+\varphi)}} \mathrm{d} \theta+\cos \varphi \int_{0}^{\theta} \frac{\cos (\theta+\varphi)}{\sqrt{K^{2}+\cos (\theta+\varphi)}} \mathrm{d} \theta\right),\left(\cos \varphi \int_{0}^{\theta} \frac{\sin (\theta+\varphi)}{\sqrt{K^{2}+\cos (\theta+\varphi)}} \mathrm{d} \theta-\sin \varphi \int_{0}^{\theta} \frac{\cos (\theta+\varphi)}{\sqrt{K^{2}+\cos (\theta+\varphi)}} \mathrm{d} \theta\right)\right]^{T}+(0, \bar{a})^{T}
\end{aligned}
$$

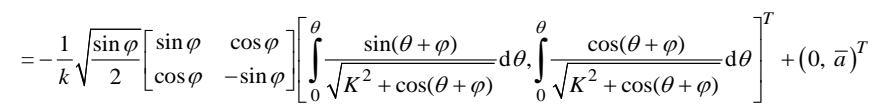

$$
\begin{aligned}
& =-\frac{1}{k} \sqrt{\frac{\sin \varphi}{2}}\left[\begin{array}{cc}
\sin \varphi & \cos \varphi \\
\cos \varphi & -\sin \varphi
\end{array}\right]\left[\begin{array}{l}
A(\theta) \\
B(\theta)
\end{array}\right]+\left(\begin{array}{l}
0 \\
\bar{a}
\end{array}\right)
\end{aligned}
$$

where

$$
\left\{\begin{array}{l}
A(\theta)=\int_{0}^{\theta} \frac{\sin (\theta+\varphi)}{\sqrt{K^{2}+\cos (\theta+\varphi)}} \mathrm{d} \theta=-2\left[\sqrt{K^{2}+\cos (\theta+\varphi)}-\sqrt{K^{2}+\cos \varphi}\right] \\
B(\theta)=\int_{0}^{\theta} \frac{\cos (\theta+\varphi)}{\sqrt{K^{2}+\cos (\theta+\varphi)}} \mathrm{d} \theta=-\sqrt{2} p\left(K^{2}\left[F\left(\frac{\theta}{2}+\frac{\varphi}{2}, p\right)-F\left(\frac{\varphi}{2}, p\right)\right]-\frac{2}{p^{2}}\left[E\left(\frac{\theta}{2}+\frac{\varphi}{2}, p\right)-E\left(\frac{\varphi}{2}, p\right)\right]\right)
\end{array}\right.
$$

\section{Appendix B:}

Replacing $\theta$ in $A(\theta)$ of Eq. (12) by $-\varphi$ and inserting $A(-\varphi)$ into the first expression of Eq. (14), we obtain,

$$
-\frac{1}{k} \sqrt{\frac{\sin \varphi}{2}}\left(-2\left[\sqrt{K^{2}+1}-\sqrt{K^{2}+\cos \varphi}\right]\right)=\bar{b}-\bar{a} \cos \varphi
$$

Re-arranging Eq. (B.1), the following expression arrives:

$$
\sqrt{K^{2}+1}-\sqrt{\frac{\sin \varphi}{2}} k \bar{b} \xi(\bar{a}, \bar{b})=\sqrt{K^{2}+\cos \varphi}
$$

Squaring both sides of the Eq. (B.2), it is re-expressed as, 


$$
1-\cos \varphi-2 \sqrt{K^{2}+1} \sqrt{\frac{\sin \varphi}{2}} k \bar{b} \xi(\bar{a}, \bar{b})+\frac{\sin \varphi}{2} k^{2} \bar{b}^{2} \xi^{2}(\bar{a}, \bar{b})=0
$$

Considering the expression of $K^{2}$ in Eq. (7), we find,

$$
\begin{aligned}
K^{2}+1 & =\frac{\sin \varphi}{2 k^{2} \bar{b}^{2}}\left(\frac{k^{2} \bar{b}^{2}}{2} \xi(\bar{a}, \bar{b})-\zeta(\bar{a}, \bar{b})\right)^{2}+1-\cos \varphi \\
& =\frac{\sin \varphi}{2 k^{2} \bar{b}^{2}}\left[\left(\frac{k^{2} \bar{b}^{2}}{2} \xi(\bar{a}, \bar{b})-\zeta(\bar{a}, \bar{b})\right)^{2}+2 k^{2} \bar{b}^{2} \frac{1-\cos \varphi}{\sin \varphi}\right] \\
& =\frac{\sin \varphi}{2 k^{2} \bar{b}^{2}}\left[\left(\frac{k^{2} \bar{b}^{2}}{2} \xi(\bar{a}, \bar{b})-\zeta(\bar{a}, \bar{b})\right)^{2}+2 k^{2} \bar{b}^{2} \xi(\bar{a}, \bar{b}) \zeta(\bar{a}, \bar{b})\right] \\
& =\frac{\sin \varphi}{2 k^{2} \bar{b}^{2}}\left(\frac{k^{2} \bar{b}^{2}}{2} \xi(\bar{a}, \bar{b})+\zeta(\bar{a}, \bar{b})\right)^{2}
\end{aligned}
$$

Substituting Eq. (B.4) into Eq. (B.3), the left side of Eq. (B.3) is expressed as,

$$
\begin{aligned}
& 1-\cos \varphi-2 \sqrt{\frac{\sin \varphi}{2}} \frac{1}{k \bar{b}}\left(\frac{k^{2} \bar{b}^{2}}{2} \xi(\bar{a}, \bar{b})+\zeta(\bar{a}, \bar{b})\right) \sqrt{\frac{\sin \varphi}{2}} k \bar{b} \xi(\bar{a}, \bar{b})+\frac{\sin \varphi}{2} k^{2} \bar{b}^{2} \xi^{2}(\bar{a}, \bar{b}) \\
= & 1-\cos \varphi-\sin \varphi\left(\frac{k^{2} \bar{b}^{2}}{2} \xi^{2}(\bar{a}, \bar{b})+\xi(\bar{a}, \bar{b}) \zeta(\bar{a}, \bar{b})\right)+\frac{\sin \varphi}{2} k^{2} \bar{b}^{2} \xi^{2}(\bar{a}, \bar{b}) \\
= & 1-\cos \varphi-\sin \varphi \xi(\bar{a}, \bar{b}) \zeta(\bar{a}, \bar{b}) \\
= & 1-\cos \varphi-(1-\cos \varphi) \\
= & 0
\end{aligned}
$$

Thus, the derivation of Eqs. (B.1-B.5) proves that the first expression in Eq. (14) always holds.

\section{Appendix C:}

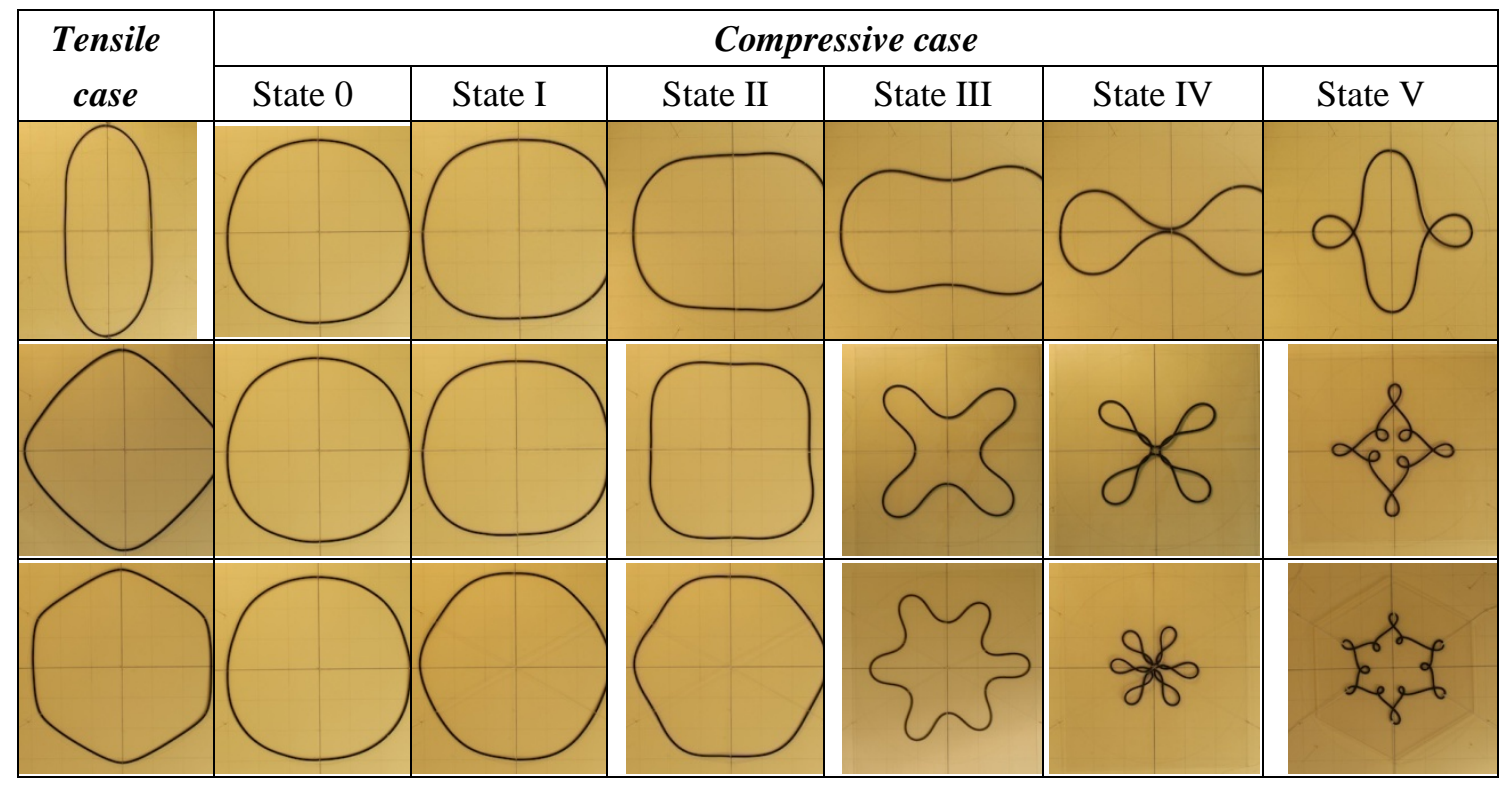




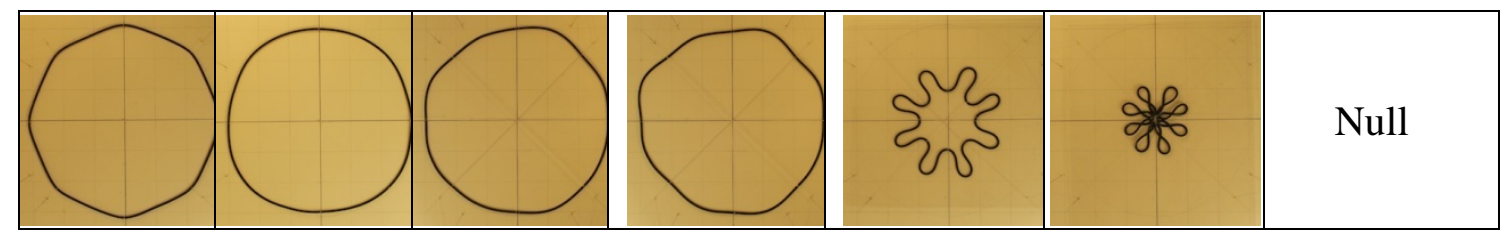

Figure S1. The patterns formed by the ring whilst being acted on by pairs of forces as described in the theoretical part of this paper. From top to bottom the pairs of forces on the ring were $N=1,2,3$ \& 4. Noted that the state $\mathrm{V}$ of the case $N=4$ is not available thanks to the complexity, and state 0 is not shown in figure 4. 


\section{References:}

[1]. Feng, C., Liew, K.W.: A molecular mechanics analysis of the buckling behavior of carbon nanorings under tension. Carbon 47, 3508-3514 (2009).

[2]. Karamanos, S.A., Eleftheriadis, C.: Collapse of pressurized elastoplastic tubular members under lateral loads. Int. J. Mech. Sci. 46(1), 35-56 (2004).

[3]. Bao, R.H., Yu, T.X.: Impact and rebound of an elastic-plastic ring on a rigid target. Int. J. Mech. Sci. 91, 55-63 (2015).

[4]. Timoshenko, S.: On the distribution of stress in a circular ring compressed by two forces along a diameter. Phil. Mag. 44, 1014-1019 (1922).

[5]. Chianese, R.B., Erdlac, R.J.: The general solution to the distribution of stresses in a circular ring compressed by two forces acting along a diameter. Q. J. Mech. Appl. Math. 41, 239-247 (1988).

[6]. Batista, M., Usenik, J.: Stresses in a circular ring under two forces acting along a diameter. J. Strain Anal. Eng. 31, 75-78 (1996).

[7]. Rao, S.S., Sundararajan, V.: In-plane flexural vibrations of circular rings. J. Appl. Mech. 36(3), 620-625 (1969).

[8]. Kirkhope, J.. In-plane vibration of a thick circular ring. J. Sound Vib. 50(2), 219-227 (1977).

[9]. Wang, C.M., Duan, W.H.: Free vibration of nanorings/arches based on nonlocal elasticity. J. Appl. Phys. 104(1), 014303 (2008).

[10].Moosavi, H., Mohammadi, M., Farajpour, A., Shahidi, S.H.: Vibration analysis of nanorings using nonlocal continuum mechanics and shear deformable ring theory. Physica E 44(1), 135-140 (2011)

[11].Chen, N., Lusk, M.T., van Duin, A.C.T., Goddard III, W.A.: Mechanical properties of connected carbon nanorings via molecular dynamics simulation. Phys. Rev. B 72(8), 085416 (2005)

[12].von Karman, T.: The buckling of thin cylindrical shells under axial compression. J. Aeronaut. Sci. 8, 303-312 (1941)

[13].Bardi, F.C., Yun, H.D., Kyriakides, S.: On the axisymmetric progressive crushing of circular tubes under axial compression. Int. J. Solids Struct. 40, 3137-3155 (2003).

[14].Marsolek, J., Reimerdes, H.G.: Energy absorption of metallic cylindrical shells with induced 
non-axisymmetric folding patterns. Int. J. Impact Eng. 30, 1209-1223 (2004).

[15].Ghosh, S.K., Johnson, W., Reid, S.R., Yu, T.X.: On thin rings and short tubes subjected to centrally opposed concentrated loads. Int. J. Mech. Sci. 23, 183-194 (1981).

[16].Reddy, T., Reid, S.: Phenomena associated with the lateral crushing of metal tubes between rigid plates. Int. J. Solids Struct. 16, 545-562 (1980).

[17].Gupta, N., Sekhon, G., Gupta, P.: Study of lateral compression of round metallic tubes. Thin Wall Struct. 43, 895-922 (2005).

[18].Olabi, A.G., Morris, E., Hashmi, M.S.J.: Metallic tube type energy absorbers: A synopsis. Thin Wall Struct. 45, 706-726 (2007).

[19].Miller, W., Smith, C.W., Scarpa, F., Evans, K.E.: Flatwise buckling optimization of hexachiral and tetrachiral honeycombs. Compos. Sci. Technol. 70, 1049-1056 (2010).

[20].Liu, J.L., Xia, R.: A unified analysis of a micro-beam, droplet and CNT ring adhered on a substrate: Calculation of variation with movable boundaries. Acta Mech. Sinica 29, 62-72 (2013).

[21].Li, R., Li, M., Su, Y., Song, J., Ni, X.: An analytical mechanics model for the island-bridge structure of stretchable electronics. Soft Matter 9(35), 8476-8482 (2013).

[22].Bisshopp, K.E., Drucker, D.C.: Large deflectionof cantilever beams. Q. Appl. Math. 3, 272-275 (1945).

[23].Elliott, J.A., Sandler, J.K., Windle, A.H., Young, R.J., Shaffer, M.S.: Collapse of single-wall carbon nanotubes is diameter dependent. Phys. Rev. Lett. 92, 095501 (2004).

[24].Pugno, N.: The design of self-collapsed super-strong nanotube bundles. J. Mech. Phys. Solids 58(9), 1397-1410 (2010).

[25].Chen, Q., Pugno, N.: Competition between in-plane buckling and bending collapses in nanohoneycombs. EPL 98, 16005 (2012).

[26].Bažant, Z.P., Cedolin, L.: Stability of structures: elastic, inelastic, fracture and damage theories. World Scientific, Singapore, pp 278-294 (2010).

[27].Guo, Y.L., Zhao, S.Y., Pi, Y.L., Bradford, M.A., Dou, C.: An experimental study on out-of-plane inelastic buckling strength of fixed steel arches. Eng. Struct. 98, 118-127 (2015).

[28].Batista, M.: A simplified method to investigate the stability of cantilever rod equilibrium 
forms. Mech. Res. Commun. 67, 13-17 (2015).

[29].Gonzalez, O., Maddocks, J.H., Schuricht, F., von der Mosel, H.: Global curvature and self-contact of nonlinearly elastic curves and rods. Calc. Var. 14(1), $29-68$ (2002).

[30].Ma, Y., Jang, K.I., Wang, L., Jung, H.N., Kwak, J.W., Xue, Y., Chen, H., Yang, Y., Shi, D., Feng, X., Rogers, J.A., Huang, Y.G.: Design of strain-limiting substrate materials for stretchable and flexible electronics. Adv. Fun. Mater. 26(29), 5345-5351 (2016). 